\title{
Simple analytical examples of boundary driven evolution of a two-dimensional magnetohydrostatic equilibrium
}

\author{
J. J. Aly
}

\author{
AIM - Unité Mixte de Recherche CEA - CNRS - Université Paris VII - UMR n 7158, Centre d'Études de Saclay, \\ 91191 Gif-sur-Yvette Cedex, France \\ e-mail: jean-jacques.aly@cea.fr
}

Received 20 July 2009 / Accepted 1 September 2009

\section{ABSTRACT}

\begin{abstract}
Aims. We construct families of time-sequences of $x$-invariant magnetostatic equilibria which describe ideal quasi-static evolutions driven by stationary shearing motions imposed on a boundary. The change in the thermal pressure of the plasma is determined by imposing either an adiabatic, or an isothermal, or an isobaric, prescription.

Methods. We start from a well known family of linear force-free fields, on which we effect simple transforms.

Results. In either case, the magnetic field and the pressure are expressed analytically as functions of space and time. The field is found to suffer an indefinite expansion, with a decrease to zero of the pressure in the adiabatic and isothermal cases, and to eventually open. Moreover, the configurations forming any sequence are shown to be linearly stable with respect to $x$-invariant perturbations.
\end{abstract}

Key words. magnetohydrodynamics (MHD) - Sun: magnetic fields - Sun: corona - Sun: coronal mass ejections (CMEs)

\section{Introduction}

Solar eruptive phenomena occurring in very elongated structures present in active regions have often been studied by using a simplified $2 \frac{1}{2} \mathrm{D}$ model. In the latter, the corona is represented by a half-space $\{z>0\}$ containing a magnetized low beta highly conducting plasma with properties independent of the $x$-coordinate. The magnetic field has an arcade topology, and it is imposed to evolve quasi-statically through a sequence of force-free configurations as a result of slow shearing motions imposed to its footpoints on the "photospheric" boundary $\{z=0\}$. Energy thus gets stored in the field, and one looks for the possibility of reaching some critical state beyond which a catastrophic release of a part of that energy becomes unavoidable. Analytical studies (Aly $1985,1990,1994)$ have shown that one of the most significant feature of such an evolution in the ideal MHD case is an indefinite expansion of the field leading asymptotically (for $t \rightarrow \infty$ ) to its partial or full opening, with the formation of an infinitely thin current-sheet, a transition by reconnection to a lower energy state becoming however energetically favorable at some stage if resistivity is introduced in the model. Numerical simulations based on both dynamical and static schemes (Amari et al. 1996; Choe \& Lee 1996) have lead to similar conclusions, and have also provided valuable descriptions of the nonideal reconnection phase.

Although the force-free assumption appears to be relevant for representing slowly evolving structures lying in the midcorona, where the plasma beta has effectively a low value, it may be much less justified to use it for studying structures extending up to the upper corona. In the latter region, pressure and gravity forces are no longer fully negligible, and it has even been suggested by several authors that they may play a crucial role in the triggering of eruptive events (e.g., Low \& Smith 1993; Shibasaki 2001). As yet these forces have been taken into account in some $2 \frac{1}{2} \mathrm{D}$ numerical simulations (Zwingmann 1987;
Finn \& Chen 1990; Choe \& Lee 1996), but not much seems to have been done from an analytical point of view (see, however, Aly 1994, in which a particular example is discussed). This has lead us to undertake a general study of the evolution of a non force-free $2 \frac{1}{2} \mathrm{D}$ equilibrium. As a first step, we have looked for exact solutions of the quasi-static evolution problem including the effects of the thermal pressure (gravity is still neglected). The aim of this paper is to present families of such solutions which seem to have not been noticed before, in spite of the fact that they can be obtained by effecting some simple transforms on a well known sequence of linear force-free solutions (described, e.g., in Priest \& Forbes 1990).

The paper is organized as follows. We first state precisely (Sect. 2) the general evolutionary problem in which we are interested. Thus we explain (Sect. 3) our method for transforming a sequence of force-free fields describing an evolution driven by a stationary shearing velocity field into a non-force-free sequence having the same property, with the pressure of the plasma evolving according to either an adiabatic prescription, or an isothermal one, or an isobaric one. This method works if the original sequence satisfies a peculiar condition, which is shown in Sect. 4 to be fulfilled by the sequence of linear force-free fields alluded to above. We can thus obtain new sequences of equilibria, whose properties are studied in Sect. 5. Our results are summarized and discussed in Sect. 6.

\section{Statement of the general problem}

\subsection{Assumptions}

We use hereafter Cartesian coordinates $(x, y, z)$. For $0<L \leq \infty$, we define $D, S$, and $\Omega$ to be, respectively, the domain $\{-L<y<$ $L ; 0<z\} \subset \mathbb{R}^{3}$, its lower plane boundary $\{-L<y<L ; z=0\}$, and its cross-section $D \cap\{x=0\}$. We assume that $D$ contains a magnetized perfectly conducting plasma whose properties are 
left invariant by the translations parallel to the $x$-axis. We denote as $\boldsymbol{B}(y, z, t)$ and $p(y, z, t)$, respectively, the magnetic field and the plasma thermal pressure at time $t$. Initially (at $t=0$ ) the system is in a given state of equilibrium, with the pressure force being balanced by the Lorentz force (the gravitational force is neglected here). The total energy (magnetic+thermal) per unit of $x$-length is finite, and $\boldsymbol{B}$ is taken to have an arcade topology and no shear $\left(B_{x} \equiv 0\right)$. In the case $L<\infty$, we also require that $B_{y}( \pm L, z, 0)=0$, i.e., the field does not thread the lateral boundaries, taken otherwise to be perfectly conducting.

For $t \geq 0$, a velocity field $\mathbf{v}(y)=v(y) \hat{\boldsymbol{x}}$ is applied to the magnetic footpoints on $S$ (also taken to be perfectly conducting), with $v(y)$ decreasing fast enough at infinity in the case $L=\infty$ for the energy input rate to keep a finite value. Consequently the system is driven into an evolution which is assumed to be quasi-static. Of course, we also need to fix a rule determining the behavior of the pressure. Hereafter, we shall use in turn the following prescriptions: (i) adiabatic prescription (Finn \& Chen 1990; Choe \& Lee 1996): the mass and the entropy contents in any flux tube are conserved, which amounts to consider the "photosphere" $S$ as a wall impeding any exchange of matter and heat between the "corona" $D$ and the subphotospheric region, while assuming that there are no heat sources or sinks in $D$. (ii) Isothermal prescription: the mass in any flux tube is conserved, while the temperature $T$ keeps a constant externally fixed value. Then mass transfer through $S$ is forbidden, while energy may be exchanged with a heat reservoir (thermostat). (iii) Isobaric prescription (Zwingmann 1987): the pressure keeps its initial value on each magnetic line. In that case, the photosphere is considered as a reservoir regulating $p$ by allowing plasma to flow into or out the corona. There does not seem to be yet an agreement on which one of these assumptions may be the most realistic for describing the corona (see the discussion in Schindler 2006). Actually, it seems likely that to obtain a definitive answer to that question it will be necessary to introduce a more global model in which both the corona and the subphotospheric layers (and then the exchange between them) are taken into account. Meanwhile, studying the consequences of our three different assumptions and comparing them is certainly a profitable exercice.

\subsection{Equations}

We use the standard representation

$\boldsymbol{B}=B_{x} \hat{\boldsymbol{x}}+\boldsymbol{B}_{p}=B_{x} \hat{\boldsymbol{x}}+\nabla A \times \hat{\boldsymbol{x}}$,

in which the field is expressed in terms of a "toroidal" function $B_{x}(y, z, t)$ and a "poloidal" flux function $A(y, z, t)$. For $A$ to be uniquely defined, we require

$A( \pm L, z, t)=0 \quad$ when $L<\infty$,
$\lim _{(|y|+z) \rightarrow \infty} A=0 \quad$ when $L=\infty$

(we can impose Eq. (2) because $B_{y}( \pm L, z, t)=0$, this condition resulting from the fact that the lateral walls are perfectly conducting and the assumption $\left.B_{y}( \pm L, z, 0)=0\right)$. The level contours $\mathcal{L}(a, t)=\{(y, z) \mid A(y, z, t)=a\}$ of $A(y, z, t)$ in $\Omega$ are the field lines of $\boldsymbol{B}_{p}$ (just note that $\boldsymbol{B}_{p} \cdot \nabla A=0$ ).

Equilibrium at each time $t$ requires $B_{x}$ and $p$ to be of the form

$B_{x}(y, z, t)=B_{x}(A(y, z, t), t)$,

$p(y, z, t)=p(A(y, z, t), t)$, and $A$ to satisfy the Grad-Shafranov equation

$-\nabla^{2} A=B_{x} \dot{B}_{x}+4 \pi \dot{p}$

where a dot denotes a derivative with respect to A at constant time (see, e.g., Schindler 2006, p. 78). Moreover, we need to have at each time $t$ (finite energy condition)

$\int_{\Omega}\left(B^{2}(y, z, t)+p(y, z, t)\right) \mathrm{d} s<\infty$.

The initial equilibrium is given, and it satisfies $B_{x}(a, 0) \equiv 0$. Its lines have an arcade topology, i.e., they connect two points of $S$ by just bridging above the so-called polarity inversion line (along which $B_{z}=0$ ). Without any loss of generality, we assume that all the lines emerge from the right side of the latter, which implies that $A(y, z, t)>0$. As a direct consequence of the frozenin law and of the velocity field on $S$ being directed along $\hat{x}$, the flux function on $S$ stays invariant,

$A(y, 0, t)=A(y, 0,0)$,

and the lines $\mathcal{L}(a, t)$ in $\Omega$ keep their initial topology,

topology $\{\mathcal{L}(a, t)\} \equiv \operatorname{topology}\{\mathcal{L}(a, 0)\}$.

It may be worth recalling that this means that $\mathcal{L}(a, t)$ is obtained from $\mathcal{L}(a, 0)$ by a continuous deformation keeping fixed the footpoints on the lower boundary of $\Omega$.

Finally, we have to write equations prescribing how $B_{x}(a, t)$ and $p(a, t)$ change with time. For that we introduce the function

$\omega(a, t):=\int_{\{A(y, z, t)>a\}} \mathrm{d} s=\int_{a}^{a_{0}}\left(\int_{\mathcal{L}\left(a^{\prime}, t\right)} \frac{\mathrm{d} l}{B_{p}}\right) \mathrm{d} a^{\prime}$,

where $a_{0}=\max _{y} A(y, 0,0)>0$, and its derivative

$\dot{\omega}(a, t)=-\int_{\mathcal{L}(a, t)} \frac{\mathrm{d} l}{B_{p}}$.

$\omega(a, t)$ represents the area of the subdomain of $\Omega$ comprised between the line $\mathcal{L}(a, t)$ and the $y$-axis, and then $-\dot{\omega}(a, t) \mathrm{d} a$ is the area of the domain comprised between the poloidal lines $\mathcal{L}(a, t)$ and $\mathcal{L}(a+\mathrm{d} a, t)$. To get an equation for $B_{x}(a, t)$, we consider a line of $\boldsymbol{B}$ admitting $\mathcal{L}(a, t)$ as its projection onto $\Omega$, and define its shear $X(a, t)$ to be the difference between the $x$-coordinates of its left and right footpoints, respectively. Then we have on the one hand $X(a, t)=\left(-B_{x} \dot{\omega}\right)(a, t)$ (just use the equation determining a magnetic line and Eqs. (4) and (11)), and on the other hand $X(a, t)=\left[v\left(y^{-}(a)\right)-v\left(y^{+}(a)\right)\right] t=: \zeta(a) t$, where $y^{ \pm}(a)$ denote the $y$-coordinates of the left $(-)$ and right $(+)$ footpoints (we use here the fact that $X(a, t)$ is created by the stationary velocity field imposed on $S$ ). Therefore $B_{x}$ has to obey the nonlocal equation

$X(a, t)=B_{x}(a, t)[-\dot{\omega}(a, t)]=t \zeta(a)$.

The pressure function $p(a, t)$ is obviously given by

$p(a, t)=p(a, 0)\left(\frac{-\dot{\omega}(a, 0)}{-\dot{\omega}(a, t)}\right)^{\Gamma}$,

where $p(a, 0)$ is its given initial value, and we select $\Gamma=0$ for an isobaric evolution, $\Gamma=1$ for an isothermal evolution, and $\Gamma=\gamma$ for an adiabatic evolution, with $\gamma>1$ being the adiabatic index of the gas. For theoretical purpose, the latter will be taken here as an arbitrary parameter.

To summarize, our problem - referred to as EvPb hereafter consists to determine a sequence $\left\{\left(A, B_{x}, p\right)(y, z, t) ; t \geq 0\right\}$ satisfying Eqs. (2)/(3)-(9) and (12), (13), when we are given an initial unsheared configuration $\left(A, B_{x}=0, p\right)(y, z, 0)$, a velocity profile $v(y)$ (and then a shear function $\zeta(a)$ ), and a value of $\Gamma$. In the case where $p(a, 0) \equiv 0, \mathrm{EvPb}$ reduces to the force-free evolutionary problem studied in the papers quoted in the introduction. 


\section{A tentative method to get solutions to EvPb having $p \neq 0$ from a force-free solution}

We assume that we know a force-free solution $\overline{\boldsymbol{B}}(t)$ of EvPb i.e., a solution for which the pressure vanishes -, and we choose a time $t_{0}>0$. Then we set for $t \geq t_{0}$

$p(a, t)=\frac{\bar{B}_{x}^{2}\left(a, t_{0}\right)}{8 \pi}\left(\frac{-\dot{\bar{\omega}}\left(a, t_{0}\right)}{-\dot{\bar{\omega}}(a, t)}\right)^{\Gamma}$,

$B_{x}^{2}(a, t)={\overline{B_{x}}}^{2}(a, t)-8 \pi p(a, t)$,

$\boldsymbol{B}(t)=B_{x}(\bar{A}(t), t) \hat{\boldsymbol{x}}+\nabla \bar{A}(t) \times \hat{\boldsymbol{x}}$,

where an overline indicates that a quantity is associated to $\overline{\boldsymbol{B}}$, and $\Gamma$ is a given number either equal to 0 , or equal to 1 , or larger than 1 . The time-sequence $(\boldsymbol{B}(t), p(t))$ that we obtain that way is a sequence of arcade equilibria as $\boldsymbol{B}$ and $\bar{B}$ have the same flux function, $\bar{A}$, and the same right-hand side of the Grad-Shafranov Eq. (6) $\left(B_{x}^{2}(a, t)+8 \pi p(a, t)={\overline{B_{x}}}^{2}(a, t)\right)$. It starts at the new initial time $t_{0}$ from the nonpotential unsheared equilibrium

$\left(\boldsymbol{B}\left(t_{0}\right), p\left(t_{0}\right)\right)=\left(\nabla \bar{A}\left(t_{0}\right) \times \hat{\boldsymbol{x}}, \bar{B}_{x}^{2}\left(\bar{A}\left(t_{0}\right), t_{0}\right) / 8 \pi\right)$,

and the thermal pressure evolves according to Eq. (13).

The shear $X(a, t)$ of the new field satisfies the first equality in Eq. (12) and then

$X^{2}(a, t)=B_{x}^{2}(a, t) \dot{\omega}^{2}(a, t)=\left[t^{2}-t_{0}^{2}\left(\frac{\dot{\bar{\omega}}\left(a, t_{0}\right)}{\dot{\bar{\omega}}(a, t)}\right)^{\Gamma-2}\right] \bar{\zeta}^{2}(a)$,

where we have used the relation $\omega(a, t)=\bar{\omega}(a, t)$. If we now suppose that the factorization

$X^{2}(a, t)=f_{t_{0}}^{2}(t) \zeta^{2}(a)$

holds true for some $\Gamma$ and some functions $\zeta(a)$ and $f_{t_{0}}(t)$, with $f_{t_{0}}\left(t_{0}\right)=0$ and $f_{t_{0}}(t)$ increasing, we can adopt $t^{\prime}=f_{t_{0}}(t)$ as a new time (as we consider a quasi-static evolution, there is no dynamics involved and time just plays the role of a labeling parameter for the configurations), and we get after substituting $f_{t_{0}}^{-1}\left(t^{\prime}\right)$ for $t$ in $(\boldsymbol{B}(t), p(t))$ a sequence $\left(\boldsymbol{B}\left(t^{\prime}\right), p\left(t^{\prime}\right)\right)$ which is clearly another solution to $\mathrm{EvPb}$.

Our "method" for constructing a solution to EvPb having $p \not \equiv$ 0 should now appear clearly. It just consists to look for force-free solutions whose function $X^{2}$ defined by Eq. (18) possesses the factorization property (19) for some value of $\Gamma$, and to apply to it the transform explained above. Obviously the method works for any $\overline{\boldsymbol{B}}(t)$ if we take $\Gamma=2$ (in that case the pressure $p$ just evolves as did the magnetic pressure ${\overline{B_{x}}}^{2} / 8 \pi$, and we can take $\zeta=\bar{\zeta}$ and $\left.f_{t_{0}}(t)=\sqrt{t^{2}-t_{0}^{2}}\right)$. That it works for other values of $\Gamma$ may appear a priori dubious. However, it turns out to be the case, as shown in the next section.

\section{An example of application of the method}

\subsection{A force-free solution to $E v P b$}

We start with a well known construction which may be found, e.g., in Priest \& Forbes (1990). We first introduce the sequence $\left\{\boldsymbol{B}_{\alpha}\right\}$ of linear arcade force-free fields defined in $\Omega$ (with $L<\infty)$ by

$\boldsymbol{B}_{\alpha}(y, z)=\nabla A_{\alpha}(y, z) \times \hat{\boldsymbol{x}}+\alpha A_{\alpha}(y, z) \hat{\boldsymbol{x}}$,

$A_{\alpha}(y, z)=\frac{B_{0}}{k} \mathrm{e}^{-z \sqrt{k^{2}-\alpha^{2}}} \cos (k y)$, where $B_{0}>0$ is a constant, $k=\pi / 2 L$, and $\alpha$ is a parameter ranging in $]-k, k$. A short calculation shows that the differential area function (see Eq. (11)) associated to $\boldsymbol{B}_{\alpha}$ is given by

$-\dot{\omega}_{\alpha}(a)=\int_{\mathcal{L}_{\alpha}(a)} \frac{\mathrm{d} l}{B_{\alpha p}}=\frac{2}{k a \sqrt{k^{2}-\alpha^{2}}} \arccos \frac{a}{a_{0}}$,

where $a_{0}=\max _{y} A(y, 0,0)=B_{0} / k$. Using the first equality in Eq. (12), we thus obtain for the shear of a line of $\boldsymbol{B}_{\alpha}$ on which $A_{\alpha}=a$

$X_{\alpha}(a)=\alpha a\left[-\dot{\omega}_{\alpha}(a)\right]=\frac{2 \alpha}{k \sqrt{k^{2}-\alpha^{2}}} \arccos \frac{a}{a_{0}}$.

Next we set for $t \geq 0$

$\bar{\alpha}(t)=k \frac{t / T}{\sqrt{1+(t / T)^{2}}}$,

where $T$ is some fixed number, and we define a time-sequence of arcade force-free fields by

$\overline{\boldsymbol{B}}(t)=\boldsymbol{B}_{\bar{\alpha}(t)}$.

For $\overline{\boldsymbol{B}}(t)$, we have

$-\dot{\bar{\omega}}(a, t)=-\dot{\omega}_{\bar{\alpha}(t)}=\frac{2 \arccos \left(a / a_{0}\right)}{k^{2} a}\left(1+\frac{t^{2}}{T^{2}}\right)^{1 / 2}$,

and the shear function writes

$\bar{X}(a, t)=X_{\bar{\alpha}(t)}=t\left[(2 / k T) \arccos \left(a / a_{0}\right)\right]=: t \bar{\zeta}(a)$.

$\bar{X}(a, t)$ is just of the form occuring in Eq. (12) and then $\overline{\boldsymbol{B}}(t)$ appears to be the solution of the EvPb defined by the initial arcade potential field $\overline{\boldsymbol{B}}(0)=\boldsymbol{B}_{\alpha=0}$, and the stationnary velocity field

$\mathbf{v}(y)=(y / T) \hat{x}$

which is easily checked to produce the shear profile $\bar{\zeta}$.

\subsection{The new solutions}

A simple look at Eq. (26) shows that the ratio $-\dot{\bar{\omega}}\left(a, t_{0}\right) /-\dot{\bar{\omega}}(a, t)$ (with $t$ and $t_{0}$ being arbitrary positive numbers) is independent of $a$, and that the term inside the bracket in the right-hand side of Eq. (18) is always positive. Then the field $\overline{\boldsymbol{B}}(t)$ has the factorization property (19) for all the values of $\Gamma$, and we can apply the method of the previous section to produce solutions to EvPb with $p \not \equiv 0$. Actually, it will prove more convenient here to start directly from the sequence $\boldsymbol{B}_{\alpha}$.

We thus fix a number $\left.\alpha_{0} \in\right]-k, k[$, and introduce the new time sequence of equilibria, $(\boldsymbol{B}(t), p(t)), t \geq 0$, by setting

$\boldsymbol{B}(t)=B_{x}\left(A_{\alpha(t)}, t\right) \hat{\boldsymbol{x}}+\nabla A_{\alpha(t)} \times \hat{\boldsymbol{x}}$,

$B_{x}(a, t)= \pm\left[\alpha^{2}(t) a^{2}-8 \pi p(a, t)\right]^{1 / 2}$,

$p(a, t)=\frac{\alpha_{0}^{2} a^{2}}{8 \pi}\left(\frac{-\dot{\omega}_{\alpha_{0}}(a)}{-\dot{\omega}_{\alpha(t)}(a)}\right)^{\Gamma}$,

where $\alpha(t)$ is an unknown function satisfying $\alpha(0)=\alpha_{0}$. This sequence starts from the unsheared equilibrium defined by

$\boldsymbol{B}(0)=\nabla A_{\alpha_{0}} \times \hat{\boldsymbol{x}} \quad$ and $\quad p(a, 0)=\frac{\alpha_{0}^{2} a^{2}}{8 \pi}$, 
with the latter having a beta at the origin given by

$\beta_{0}=\frac{p(0,0,0)}{B^{2}(0,0,0) / 8 \pi}=\frac{\alpha_{0}^{2}}{k^{2}-\alpha_{0}^{2}} \quad \Rightarrow \quad \frac{\alpha_{0}^{2}}{k^{2}}=\frac{\beta_{0}}{1+\beta_{0}}$.

Using Eqs. (12), (22), and (30) we get the relation

$X^{2}(a, t)=\left[\alpha^{2}(t)-\alpha_{0}^{2}\left(\frac{k^{2}-\alpha^{2}(t)}{k^{2}-\alpha_{0}^{2}}\right)^{\Gamma / 2}\right] \frac{\left[2 \arccos \left(a / a_{0}\right)\right]^{2}}{k^{2}\left(k^{2}-\alpha^{2}(t)\right)}$

for the shear $X(a, t)$. The latter can be put into the form $X(a, t)=$ $t \zeta(a)$ by choosing $\zeta(a)$ to be of the form (27) - the shearing velocity field thus keeping the form (28), with $T$ having the sign of $B_{x}$ - and by requiring $\alpha^{2}(t)$ to be a solution of the equation

$\frac{t^{2}}{T^{2}}=\frac{\alpha^{2}(t)}{k^{2}-\alpha^{2}(t)}-\frac{\alpha_{0}^{2}}{\left(k^{2}-\alpha_{0}^{2}\right)^{\Gamma / 2}\left(k^{2}-\alpha^{2}(t)\right)^{1-\Gamma / 2}}$.

Simple considerations show that Eq. (35) has a unique solution $\alpha^{2}\left(t, \alpha_{0}^{2}, \Gamma\right)$ for $t \geq 0$, with the latter satisfying

$\frac{\partial \alpha^{2}}{\partial t}>0, \frac{\partial \alpha^{2}}{\partial \alpha_{0}^{2}}>0, \frac{\partial \alpha^{2}}{\partial \Gamma}<0$.

Then $\alpha^{2}$ increases with $t$ (actually from $\alpha_{0}^{2}$ up to $k^{2}$ ) and with $\alpha_{0}^{2}$, while it decreases with $\Gamma$.

In many cases, it is possible to give $\alpha^{2}$ in closed form:

- For the isobaric law, $\Gamma=0$, we have

$$
\alpha^{2}\left(t, \alpha_{0}^{2}, 0\right)=\frac{\alpha_{0}^{2}+k^{2} t^{2} / T^{2}}{1+t^{2} / T^{2}} .
$$

- For the isothermal law, $\Gamma=1$, we can write

$$
\begin{aligned}
& \alpha^{2}\left(t, \alpha_{0}^{2}, 1\right)=k^{2}-\frac{1}{4\left(1+t^{2} / T^{2}\right)^{2}} \\
& \quad \times\left\{\left[\frac{\alpha_{0}^{4}}{k^{2}-\alpha_{0}^{2}}+4 k^{2}\left(1+\frac{t^{2}}{T^{2}}\right)\right]^{1 / 2}-\frac{\alpha_{0}^{2}}{\left(k^{2}-\alpha_{0}^{2}\right)^{1 / 2}}\right\}^{2} .
\end{aligned}
$$

- For the adiabatic law, $\Gamma=\gamma>1$, a closed form solution is possible for the particular values of $\gamma$ for which Eq. (35) reduces to an algebraic equation of degree no larger than 5 ( $\gamma=$ $6 / 5,8 / 5,4 / 3,3 / 2,2,5 / 2,8 / 3,3,10 / 3,4,5,6,8,10)$. For instance, we obtain in the simplest case where $\gamma=2$

$\alpha^{2}\left(t, \alpha_{0}^{2}, 2\right)=\frac{\alpha_{0}^{2}+\left(k^{2}-\alpha_{0}^{2}\right) t^{2} / T^{2}}{k^{2}+\left(k^{2}-\alpha_{0}^{2}\right) t^{2} / T^{2}} k^{2}$.

We shall refrain here from writing the quite heavy formulas for the other values of $\gamma$ as they are not very illuminating.

\section{Some properties of the new solutions}

\subsection{Expansion of the poloidal structure}

It results immediately from Eq. (21) that the maximum height, $Z(a, t)$, reached at time $t$ by the line $\mathcal{L}(a, t)$ is given by

$Z(a, t)=\frac{1}{\sqrt{k^{2}-\alpha^{2}(t)}} \ln \frac{a_{0}}{a}$.

When $t$ increases from 0 to infinity, $\alpha^{2}(t)$ increases from $\alpha_{0}^{2}$ up to $k^{2}$, and then $Z(a, t)$ increases monotonically up to infinity, with an asymptotically constant speed. Then shearing the footpoints
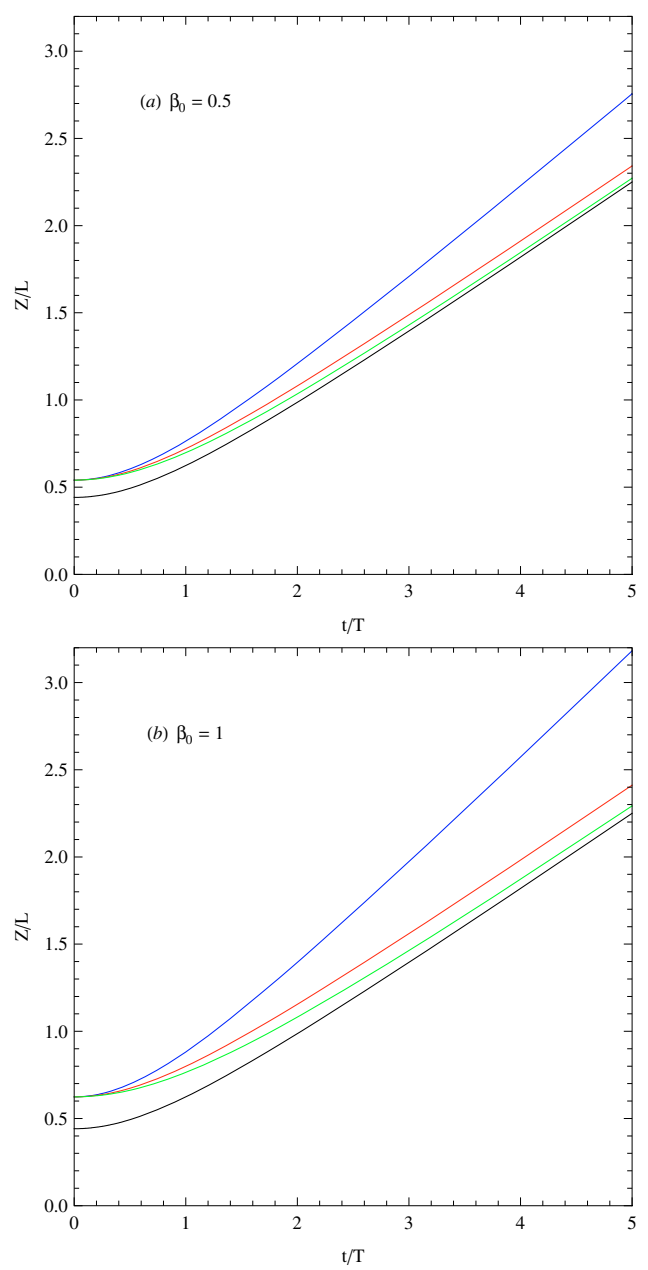

Fig. 1. Evolution of $Z\left(a_{0} / 2, t\right) / L$ as a function of $t / T$ for $\beta_{0}=0.5$ a) and $\beta_{0}=1.0 \mathbf{b}$ ). In both $\mathbf{a}$ ) and $\mathbf{b}$ ), we have plotted (from the bottom to the top) the force-free case $\left(\beta_{0}=0\right.$, black curve), and the three cases $\Gamma=2$ (green), $\Gamma=1$ (red), and $\Gamma=0$ (blue).

leads to a continuous expansion of the poloidal structure and to its eventual opening. Moreover, it results from Eq. (36) that increasing $\alpha_{0}^{2}$ leads to an increase of $Z(a, t)$ while increasing $\Gamma$ leads to a decrease of that quantity. This behavior is illustrated in Fig. 1 where we have plotted $Z(a, t) / L$ for $a=a_{0} / 2, \Gamma=0,1,2$, and $\alpha_{0}^{2}$ such that $\beta_{0}=0.5,1$ (see Eq. (33)).

The open equilibrium which is asymptotically approached when $t \rightarrow \infty$ is $1 \mathrm{D}$ for $\Gamma \geq 1$, being given by

$\boldsymbol{B}(\infty)=B_{0}[\cos (k y) \hat{\boldsymbol{x}}+\sin (k y) \hat{z}]$,

$p(\infty)=0$,

and $1 \frac{1}{2} \mathrm{D}$ for $\Gamma=0$, in which case

$\boldsymbol{B}(\infty)=B_{0}\left[\left(1-\alpha_{0}^{2} / k^{2}\right)^{1 / 2} \cos (k y) \hat{\boldsymbol{x}}+\sin (k y) \hat{\boldsymbol{z}}\right]$,

$p(\infty)=\frac{\alpha_{0}^{2} B_{0}^{2}}{8 \pi k^{2}} \cos ^{2}(k y)$.

In these final states, the total pressure $P$ (magnetic+thermal) is uniform $\left(P=B_{0}^{2} / 8 \pi\right)$, but no current sheet is present (this is a peculiar feature due to their $1 / 1 \frac{1}{2} \mathrm{D}$ character).

\subsection{Energy}

Let us denote by $W_{p}, W_{x}$, and $W_{i}$, respectively, the poloidal magnetic energy (energy of $\boldsymbol{B}_{p}$ ), the toroidal magnetic energy 
(energy of $B_{x}$ ), and the plasma internal energy (of course "energy" means here "energy per unit of $x$-length"). Then we get after a short calculation

$W_{p}(t)=\frac{B_{0}^{2}}{32 k^{3}} \frac{2 k^{2}-\alpha^{2}(t)}{\sqrt{k^{2}-\alpha^{2}(t)}}$,

$W_{x}(t)=\frac{B_{0}^{2}}{32 k^{3}} \sqrt{k^{2}-\alpha^{2}(t)} \frac{t^{2}}{T^{2}}$,

$W_{i}(t)=\frac{B_{0}^{2}}{32(\gamma-1) k^{3}} \frac{\alpha_{0}^{2}}{\sqrt{k^{2}-\alpha^{2}(t)}}\left(\frac{k^{2}-\alpha^{2}(t)}{k^{2}-\alpha_{0}^{2}}\right)^{\Gamma / 2}$,

and the total energy $W=W_{p}+W_{x}+W_{i}$ is given by

$W(t)=\frac{B_{0}^{2}}{32 k^{3} \sqrt{k^{2}-\alpha^{2}(t)}}\left[2 k^{2}+\alpha_{0}^{2} \frac{2-\gamma}{\gamma-1}\left(\frac{k^{2}-\alpha^{2}(t)}{k^{2}-\alpha_{0}^{2}}\right)^{\Gamma / 2}\right]$.

As it is easily checked, $W_{p}, W_{x}$, and $W$, increase monotonically with time in any case, and they all tend to infinity when $t \rightarrow$ $\infty$, while $W_{i}$ increases up to infinity in the isobaric case $(\Gamma=$ $0)$, keeps a constant value in the isothermal case $(\Gamma=1)$, and decreases to zero in the adiabatic case $(\Gamma>1)$.

\subsection{Linear stability}

Consider any one of the configurations forming a solution to $\mathrm{EvPb}$, and submit it - the driving boundary motions being frozen - to an arbitrary small $2 \frac{1}{2} \mathrm{D}$ displacement field $\xi(y, z)=$ $\xi_{x}(y, z) \hat{\boldsymbol{x}}+\xi_{p}(y, z)$. We require $\xi$ to satisfy the conditions

$\xi(y, 0)=0 \quad$ and $\quad \xi_{y}( \pm L, z)=0$,

which express the fact that the boundary of $D$ is rigid and perfectly conducting. Then we claim that the configuration is ideally linearly stable with respect to this perturbation. This statement is an immediate consequence of a general result reported in Schindler (2006, p. 216). According to the latter, a $2 \frac{1}{2} \mathrm{D}$ configuration in $\Omega$ is stable if there does exist a fixed direction $\hat{\boldsymbol{u}}$ such that $\hat{\boldsymbol{u}} \cdot \boldsymbol{B}_{p}$ keeps the same sign in the whole domain. This is clearly the case here, with $\hat{\boldsymbol{u}}=\hat{\boldsymbol{y}}$, as we have $\hat{\boldsymbol{y}} \cdot \boldsymbol{B}_{p}=B_{y}<0$.

\section{Conclusion}

Up to now the analytical problem of the boundary driven quasistatic evolution of an $x$-invariant magnetostatic equilibrium occupying either a half-space or a vertical slice of it has been essentially considered in the case where the magnetic field is force-free. In this paper, we have proposed a method which allows to construct examples in which the thermal pressure of the plasma is taken into account. The method amounts to transform a solution of a force-free $\mathrm{EvPb}$ into a solution of a non-force-free $\mathrm{EvPb}$, with $p$ evolving according to some prescribed law, either adiabatic, or isothermal, or isobaric. For the method to apply, the initial force-free solution has however to obey a strong peculiar constraint. The latter has been checked to be satisfied by a well known linear force-free solution which has been already used by many authors, and we have explicitly constructed from it solutions to EvPb driven by the same boundary velocity field, under each one of the three prescriptions for $p(t)$ recalled above. In the case where the constraint is not satisfied, the transform may be still effected, but it leads to evolutionary sequences which are driven by boundary motions whose velocity profile evolves in time and becomes in any case dependent of the imposed pressure prescription. Unfortunately, the transform from a force-free state to a non-force-free one which is at the base of the method is specific to the class of translation-invariant equilibria, for which the thermal pressure and the magnetic pressure associated to the component of the field along the direction of invariance intervene on the same footing in the Grad-Shafranov equation. This property is no longer true for either the axisymmetric or the helical equilibria, and a fortiori for the $3 \mathrm{D}$ ones.

It should be noted that many authors have already provided examples of conversion of some particular magnetostatic equilibrium into a new one as a result of the application of an adequate mathematical transform (e.g., Low 1982; Lites et al. 1995; Aly 2009). For instance, Low 1982 has shown that any one of the specific unsheared 2D equilibria we have used in Sect. 4.2 as possible initial states of a quasi-static evolution, can be transformed into a 3D laminar equilibrium submitted to a uniform gravitational field. The new configuration consists of discrete, finite-thickness flux tubes embedded in an isothermal field-free atmosphere, and it turns out to be linearly stable with respect to 3D perturbations. There is however something new in the present paper: the transform method has been applied not to a single equilibrium at a time, but to a whole evolutionnary sequence driven by stationary boundary motions, with another sequence of the same type being eventually obtained.

One of the weakness of the solutions we have presented is of course the presence of the lateral walls which impose to the field an artificial confinement, and it is necessary to study in details the more interesting case where the evolution takes place in the whole half-space $(L=\infty)$. Preliminary results on that problem show that - as it can be a priori expected - the pressure does not change qualitatively the evolution when either the adiabatic or the isothermal prescription is adopted. In the isobaric case, however, the evolution cannot go on for ever. There is a critical time at which a global nonequilibrium phenomenon (Aly 1993) develops: there is no longer an equilibrium compatible with the constraints imposed to the system, and a dynamical evolution has to start in, which may be guessed to lead to an opening of the field. In spite of that, we feel that the new solutions are quite useful. They give explicit examples in which it is possible to evaluate quantitatively how a force-free solution is changed when the effects of the thermal pressure of the plasma are introduced, they give an interesting test for checking the accurateness of the various exact estimates (generally in the form of upper and lower bounds on some physical quantities) that we shall present in our forthcoming paper on the general problem, and finally they may be used as test cases for numerical MHD codes of evolution.

\section{References}

Aly, J.-J. 1985, A\&A, 143, 19

Aly, J.-J. 1990, Comp. Phys. Comm., 59, 13

Aly, J. J. 1993, in Cosmical Magnetism, ed. D. Lynden-Bell (Cambridge: Institute of Astronomy), 7

Aly, J. J. 1994, A\&A, 288, 1012

Aly, J.-J. 2009, preprint

Amari, T., Luciani, J.-F., Aly, J.-J., \& Tagger, M. 1996, A\&A, 306, 913

Choe, G. S., \& Lee, L. C. 1996, ApJ, 472, 360

Finn, J. M., \& Chen, J. 1990, ApJ, 349, 345

Lites, B. W., Low, B. C., Martinez Pillet, V., et al. 1995, ApJ, 446, 877

Low, B. C. 1982, ApJ, 263, 952

Low, B. C., \& Smith, D. F. 1993, ApJ, 410, 412

Priest, E. R., \& Forbes, T. G. 1990, Sol. Phys., 130, 399

Schindler, K. 2006, Physics of Space Plasma Activity (Cambridge: Cambridge University Press)

Shibasaki, K. 2001, ApJ, 557, 326

Zwingmann, W. 1987, Sol. Phys., 111, 309 